\title{
Jessica Nowoczien (2012): Drama in the Classroom.
}

\author{
Dramenarbeit im Englischunterricht der Sekundarstufe I im \\ Hinblick auf Gendersensibilisierung und interkulturelle \\ Kommunikation. Frankfurt a. M.: Peter Lang. ISBN: \\ 978-3-631-62069-4.
}

\section{Stefanie John}

Lassen sich klassische Dramentexte mit der Lebenswelt von Jugendlichen im 21. Jahrhundert verknüpfen? Welche Rolle kann das Medium Theater im Englischunterricht der Sekundarstufe I einnehmen? Diese Fragen bilden den Ausgangspunkt für das Dissertationswerk Jessica Nowocziens, das sich eine praxisbezogene Verknüpfung der Kategorien Gender und Interkulturalität anhand von Theaterarbeit im Fremdsprachenunterricht zum Ziel setzt. Herzstück der Arbeit ist ein komplexer Unterrichtsentwurf zu ausgewählten Dramen William Shakespeares. Weit über eine reine inhaltliche und formale Textanalyse hinausgehend soll hier die theaterpraktische Methodik Schüler und Schülerinnen für eine Auseinandersetzung mit Geschlechterrollen und kulturellen Identitäten sensibilisieren. Ein ambitioniertes Projekt, das die vorliegende über 300 Seiten lange Monografie zunächst ausführlich theoretisch kontextualisiert und dann in seinem praktischen Verlauf beschreibt und bewertet. Dabei überzeugt der Praxisteil insgesamt mehr als die etwas oberflächlich gestaltete theoretische Verankerung in Theorien der Geschlechterforschung.

$\mathrm{Zu}$ Recht wirft die Autorin einleitend die Frage auf, wie die Institution Schule auf die Pluralität kultureller Lebensformen und die rasanten Wandlungsprozesse westlicher Gesellschaften im 21. Jahrhundert angemessen reagieren kann und soll. Ihr Forschungsprojekt sieht die Antwort in der Zusammenführung von Dramapädagogik und Fremdsprachenunterricht. Die Forschungsübersicht der Arbeit veranschaulicht zunächst die zunehmende Relevanz, die im deutschsprachigen Raum dem darstellenden Spiel als Medium der Förderung kommunikativer Kompetenzen zumindest in der Theorie beigemessen wird. Jedoch beziehen sich solche Studien meist auf den schulischen Sekundarbereich II. Eine Prüfung der Effektivität dramenpädagogischer Konzepte im Rahmen des fremdsprachlichen Literaturunterrichts in der Sekundarstufe I bleibt laut der Autorin bisher aus. Auch seien interdisziplinäre Ansätze, die gezielt Grenzen zwischen Sprachunterricht und darstellerischen Methoden verwischen, in den bestehenden schulischen Strukturen in Deutschland längst nicht ausreichend etabliert. 
Die Vorteile dieser Methodik liegen jedoch auf der Hand, argumentiert Nowoczien. So fördere das Medium Theater durch „,seine Aufforderungen zum Spiel“, „seinen Dialogcharakter" (83) und seine Unmittelbarkeit nicht nur den authentischen Sprachgebrauch und interkulturelle Kompetenz, sondern lässt darüber hinaus einen individuellen, auf persönlichen Erfahrungen beruhenden Zugang zu komplexen Texten und Themen zu. Am Beispiel Shakespeare will die Autorin dies demonstrieren. Im Laufe eines mehrwöchigen Unterrichtsexperiments, das Nowoczien selbst durchführte, sollte ein Englischkurs des neunten Jahrgangs einer Gesamtschule eigenständig ein Shakespeare-Drama inszenieren. Dabei galt es, den Lernenden anhand von kreativen darstellerischen Übungen einen individuellen Zugang zum Text zu verschaffen.

Der Innovationsgehalt des vorgestellten Unterrichtsmodells liegt allerdings in der abstrakten Funktion, die die Theaterpraxis erfüllen soll, nämlich Jugendliche zum reflektierten Umgang mit geschlechterbasierten und kulturellen Rollenmustern zu bewegen. In der theoretischen Einführung erläutert die Autorin zunächst die Bedeutung der Aspekte Gender und Interkulturalität für den Fremdsprachenunterricht. Basierend auf etablierten Theoriemodellen von u.a. Michael Byram wird betont, dass Sprachenlernen über die Imitation des Muttersprachlers hinaus gehen und einen erfolgreichen Kommunikationsfluss, ungeachtet sprachlicher Feinheiten, fördern sollte. Gender und Interkulturalität betrachtet die Autorin als geeigneten Anhaltspunkt für dieses Vorgehen,

sind dies doch zwei wesentliche Persönlichkeitsmerkmale, über die sich ein Sprecher bzw. eine Sprecherin in der fremdsprachlichen Kommunikation vordergründig auch definieren wird(5).

Neben neueren Ansätzen der Fremdsprachendidaktik beruft sich die Arbeit stark auf aktuelle Trends in der Kultur- und Literaturtheorie. So werden im Zuge des cultural turn und der poststrukturalistischen Theoriebildung Kategorien wie Gender und Kultur bzw. Identität als flexible Konstruktionen gelesen, die in den pluralistischen Gesellschaftsformen unserer Zeit in einem dynamischen Verhältnis zueinander stehen:

Gender und Kultur [...] bedingen und beeinflussen einander fortlaufend, sodass es weder Universalität noch Eindeutigkeit oder Kontinuität einer bestehenden Identität geben kann(37).

Nowocziens didaktische Verknüpfung dieser Kategorien strebt an, gerade junge Lernende, die sich im Zuge des Fremdsprachenunterrichts bereits ganz automatisch mit Selbst- und Fremdwahrnehmungen auseinandersetzen, für diese Vielfalt und den performativen Charakter von Identitäten zu sensibilisieren. Dabei sollen Shakespeare und Theorien der Geschlechterforschung helfen.

$\mathrm{Zu}$ diesem Zweck definiert Nowoczien ,Gender ${ }^{6}$ - in der Tradition der modernen Gender Studies und Queer Theory - nach Judith Butlers Konzepts der Performativität. Es wird prägnant erläutert, wie sich Gender als eine performativ im gesellschaftlichen ,Rollenspiel‘ erzeugte, fiktive Kategorie 
verstehen lässt, die körperliche Eigenschaften nicht mehr als Ausgangspunkt für Geschlechtsidentität und Begehrensstrukturen annimmt.

Hier möchte Nowoczien eine Brücke von der Theorie zur Unterrichtspraxis schlagen. Anhand der Theatermethodik versucht sich die Arbeit an einer praktischen, in der Tat,performativen' Umsetzung theoretischer Konzepte, die Butler und ihre KollegInnen kaum zu leisten vermögen. Leider bleibt der angekündigte Rückgriff auf die Gender Studies und Queer Theory jedoch recht oberflächlich, da sich insbesondere der überblicksartige Abschnitt zur Relevanz der Queer Theory fast ausschließlich auf Sekundärliteratur und Einführungswerke beruft. Außer Butler werden überhaupt keine weiteren richtungsweisenden AutorInnen dieser Theorieschule (z.B. Sedgwick, Sinfield) einbezogen - obwohl gerade Shakespeares Dramen längst etablierter Bezugspunkt für die Queer Theory sind.

Die Besonderheit der Arbeit macht jedoch weniger die theoretische Kontextualisierung, sondern vielmehr der zweite, praxisbezogene Teil aus. Die Verlaufsbeschreibung und kritische Reflexion des Unterrichtsprojekts - ausgewertet anhand der Methode der teilnehmenden Beobachtung - bieten viele Denkanstöße für FremdsprachenlehrerInnen. So präsentiert die Arbeit einen fundierten Überblick zu etablierten Techniken des Darstellenden Spiels (Rollenspiel, Improvisation, Standbilder, hot seating u.a.) und anderen kreativen Verfahren der Texterschließung, die zum Ausprobieren einladen. Bei dem Shakespeare-Projekt gilt es, „auch methodisch eine größtmögliche Offenheit zu demonstrieren" (157). So wurde bereits in der warming up- Phase der spielhafte Charakter der Theaterarbeit genutzt, um schließlich die SchülerInnen gruppenweise in Eigenregie jeweils ein Drama inszenieren zu lassen.

Der Fokus liegt hier auf der individuellen Annäherung der Lernenden zum literarischen Text. So argumentiert die Autorin, dass gerade Shakespeares Dramen - die ihren SchülerInnen allerdings nur in verkürzter und stark vereinfachter Version zugänglich gemacht wurden - zeitgemäße Thematiken, interessante Charaktere und nicht zuletzt dramatische Spannung kombinieren, die auch Jugendliche unseres Medienzeitalters ansprechen. Darstellerische Übungen mit individuellem, lebensweltnahen Zuschnitt sollen dabei zur Auseinandersetzung mit Shakespeare motivieren:

Die Jugendlichen sollten je nach persönlichem Interesse ihre eigenen Themenschwerpunkte wählen. Individualisierte affektive Textarbeit sollte als Schwerpunkt gesehen werden und nicht etwa eine vornehmlich inhaltliche angeleitete Auseinandersetzung mit identitätskritischen Diskursen(152).

Als Ausgangspunkt dienten sensibilisierende Übungen (z.B. das Verfassen sogennanter name-stories), bei denen die Lernenden anhand ihrer persönlichen Biografien die Zusammengesetztheit der eigenen Identität im Hinblick auf Gender und kulturellen Hintergrund erkennen sollen. Nowoczien beschreibt eingehend, wie sich besonders bei den Schülerinnen ein starkes Mitteilungsbedürfnis über ihre persönliche Selbst- und Fremdwahrnehmung manifestiert.

Während der eigentlichen Inszenierungsphase sollten diese Einsichten dann auf die Dramentexte angewandt werden - bzw. zur Erschließung des litera- 
rischen Materials dienen. Dass die erhoffte individuelle Schwerpunktsetzung in Nowocziens Unterrichtsversuch zumindest bei einigen Schülergruppen gelang, zeigen kreative Endergebnisse wie eine Schülerproduktion mit dem vielsagenden Titel Romeo and Juliet in da Ghetto.

Der literarische Text verliert bei dieser Herangehensweise seinen traditionellen Hoheitsstatus:

Es gilt also Shakespeare zu entmystifizieren und den elitären Charakter, der sich mit dem Studium seiner Texte verwoben hat, abzustreifen um seine Werke auch für die Sekundarstufe I nutzbar zu machen(154).

Wenn diese Form der Produktivmachung von Literatur hier auf die Jugendlichen auch höchst motivierend wirkte (eine Schülerin Nowocziens bezeichnet das Projekt abschließend als „one of the best projects I've ever done“ (299)), so ist es dennoch dahingestellt, wie genau NeuntklässlerInnen die Textvorlage eigenständig zu verstehen vermögen. Bei der Lektüre von Nowocziens Ausführungen fragt man sich bald, ob es überhaupt noch Sinn macht, Shakespearedramen zum Unterrichtsgegenstand zu machen, wenn jeglicher kulturhistorischer Kontext, die subtile sprachliche Ironie und politische Brisanz der Texte wegfällt und sie nur auf Spannungsbögen und universelle, simplere Themen wie ,Liebe' oder ,Vater-Tochter-Beziehungen' reduziert werden. Diese finden sich zugegebenermaßen auch in jedem modernen Jugendroman oder -drama. ${ }^{1}$

Im Falle des vorgestellten Projekts drängt sich daher immer wieder die Frage der realistischen Durchführbarkeit auf. So werden von den Jugendlichen nicht nur kreative Inszenierungsideen, sondern auch ein eigenständiges Verständnis des literarischen Texts abverlangt. Jede noch so freie Inszenierungsarbeit setzt schließlich ein genaues Einarbeiten in die Textvorlage voraus. In der vorliegenden Projektbeschreibung findet der Leseprozess allerdings kaum Platz.

Bei der Schülergruppe, mit der Nowoczien ihr Projekt durchführte, handelte es sich zudem um einen leistungsstarken Kurs, in dem die meisten SchülerInnen bereits Vorerfahrung im Fach Darstellendes Spiel besaßen. Wie die Autorin abschließend selbst einräumt, kann ihr Projekt also keineswegs als repräsentativ, sondern lediglich als ein Muster für die theaterpraktische Herangehensweise an literarische Texte in der Sekundarstufe I gewertet werden, das im Falle einer anderen Lerngruppe vollkommen andere Ergebnisse erzielen könnte. Nichtsdestotrotz betont Nowoczien den großen Mehrwert theaterpraktischer Methoden im Fremdsprachen- und Literaturunterricht, der in ihrer Adaptierbarkeit für die Lebenswelt von Jugendlichen besteht:

Es kann kein Zweifel mehr daran bestehen, dass darstellendes Spiel eine wertvolle Methode ist Jugendlichen in ihrer Individualität zu begegnen"(309)

\footnotetext{
${ }^{1}$ Die Kontroverse um die Unantastbarkeit des literarischen Texts einerseits und seine Funktionalisierung für didaktische Zwecke andererseits ist in der Didaktikforschung keineswegs neu. Vgl. etwa die als Haas-Hurrelmann-Debatte bekannt gewordene Diskussion zur Funktion von Kinder- und Jugendliteratur in Praxis Deutsch 89/1988-94/1989.
} 
- so das Fazit der Autorin.

Auch wenn die Verknüpfung von Theorie- und Praxisteil der Monografie nur zum Teil überzeugt, ist die gesamte Arbeit doch aus wissenschaftlicher wie schulpraktischer Sicht durchaus empfehlenswert. Nicht zuletzt lassen sich die hier erprobten Unterrichtsideen, die Jugendlichen die Rollenhaftigkeit von Identitätsmustern vor Augen führen, vielfältig einsetzen und beispielsweise auch zur Prävention von Gewalt und Mobbing im Schulalltag nutzen. 